\title{
Comparative study between interference screw fixation and implant free technique in anterior cruciate ligament reconstruction using semitendinosus and gracilis auto graft By
}

\author{
Mohamed Ali Ahmed, Ashraf RashadMarzouk, Abdelrahman Hafez Khalifa, \\ AnisShiha
}

\begin{abstract}
Introduction to compare between interference screw fixation and implant free technique in anterior cruciate ligament reconstruction using semitendinosus and gracilis auto graft. Patients and methods this prospective study included 60young active patients. There were 56 males and 4 females. The mean age was27 years (range20-35). The meanfollowup was 24 months (range 12-48). Patients were evaluated using subjective as well as objective International KneeDocumentation Committee (IKDC) assessment forms, ResultsAssessment using IKDC scoring revealed that $90 \%$ of the patients had normal or nearly normal knee (IKDC score of $\mathbf{A} / \mathbf{B}$ ). Postoperative level of activity was excellent in $85 \%$ of patients.

ConclusionThis study showed that implant free technique in anterior cruciate ligament reconstruction using semitendinosus and gracilis auto graft

ACL reconstruction is comparable to interference screw fixation in clinical out come

Keywords Arthroscopic .ACL.Implantfree.Interferencescrew. Reconstruction.Press.Fit .graft

\section{Introduction}

The anterior cruciate ligament (ACL) is essential to the normal function of the knee, and it is oneof the most frequently injured ligaments in the human body. Its injury affects knee stability, which may causegiving way symptoms, increased risk of meniscal injuries and early onset of joint degeneration [1].

When treating an injured ACL, many decisions must be taken in consideration, especially if surgery is to be performed. The decision regarding graft choice and its fixation remains one of the most controversial. The graft could be auto

graft, allograft, or synthetic. These include patellar tendon, hamstring tendons, quadriceps tendon and others [2].

Central third bone-patellar tendon-bone auto graft fixed with interference screws has long been the graft of choice [3], despite certain number of various complications that have been reported [4].To avoid disadvantages related to internal fixationdevices, especially on femoral side, a hardware-free ACLreconstruction technique was developed. This techniqueuses the bone
\end{abstract}


SOHAG MEDICAL JOURNAL implant free technique

Vol. 21 No.1 Jan 2017

plugs on either end of the patellar tendongraft for press-fit fixation. The presented technique wasoriginally developed in $\mathbf{1 9 8 7}$ for femoral press-fit fixationand in $\mathbf{1 9 8 9}$ for tibial press-fit fixation [5]. After-wards it was used and popularized by other authors [6]. The press-fit fixation was reported to have a similar

Pull-out strength and stiffness when compared to hard-ware fixations [7] and accepted as an effective and cost reducing method for ACL reconstruction. The aim of this study is to Compare between interference screw fixations and implant free technique in anterior cruciate ligament reconstruction using semitendinosus and gracilis auto graft

\section{Patients and methods}

Prospective clinical study was conducted from January 2013 to December 2015 in our department, it included 60 patients, 56 males and 4 females, $\mathbf{3 0}$ patients under gone interference screw fixation while the other 30 patients under gone implant free technique ) average age was 27years (20- 35years). Inclusion criteria were age below $\mathbf{3 5}$ years, presence of clinical, radiological, and arthroscopic evidence of ACL deficiency, persistent symptoms after adequate conservative treatment, active, motivated patients involved in vigorous activities, good quadriceps strength, active full range of motion ROM with no extensor lag. Exclusion criteria were patients with other systemic diseases compromising their pre-
Mohamed Ali Ahmed

anesthetic fitness, patients with associated fractures of the same lower limb or spine, adolescents with open physes, , patients with local skin infections, and patients having remote infection that might have seeded in the joint.

\section{Surgical technique}

Under spinal anesthesia. Diagnostic arthroscopy is performed and any associated Chondral lesions or meniscal tears are diagnosed and treated. The ACL stump is debrided using shaver, semitendinosus and gracilis tendons are harvested and the graft is prepared on the back table Femoral tunnel is drilled using a guide wire through the anteromedial portal with free hand technique, we introduce the mosicoplasty knife according to the desired graft size, the knife is hammered to the planned depth; 20-30 $\mathrm{mm}$, then rotated to harvest the bone plug from the femur in $\mathbf{3 0}$ patients while the other $\mathbf{3 0}$ patients we use ACLfemoral drill bite (diameter according to the graft size), all these steps done while the knee is flexed at $\mathbf{1 2 0}$ degree the endoscopic aimer for the tibial tunnel is adjusted to $\mathbf{5 5}^{\circ}$ position, and the guide-tip is positioned Intra-articular through the anteromedial portal. We plan the intraarticular tibial insertion of ACL in $\mathbf{5}$ $\mathrm{mm}$ anterior to the PCL attachment towards the anterior horn of medial meniscus. The mosaicoplasty knife is used to harvest the bone plug from the tibial tunnel in $\mathbf{3 0}$ patients while the other 30 patients we use the tibial drill bite according to the graft diameterGraft 
SOHAG MEDICAL JOURNAL Comparative study between interference screw fixation and implant free technique

Vol. 21 No.1 Jan 2017

Mohamed Ali Ahmed

passage Using the suture retriever passing through the tibial tunnel we pull the distal end of the double looped suture, now the double looped suture pass through the tibial tunnel, the knee joint and the femoral tunnel, the double looped graft is folded over the double looped suture and the proximal end of the suture is pulled to allow passage of the graft through the tibial and femoral tunnel from down upwards.. After that we do small medial Para patellar incision to allow visualization of the femoral tunnel and apply traction on the proximal end of the graft while we fix it by the bone plug previously harvested from the femoral tunnel using press fit technique. then we extend knee joint to about 30 degree flexion and do tensioning of graft and fix the tibial part by the harvested tibial bone plug and then assess knee stability and tensioning of the graft in $\mathbf{3 0}$ patients while the other 30 patients we use interference screws to fix the tendon graft in both femoral and tibial side. Wound closure: After irrigation of the knee joint, the subcutaneous tissue and skin are closed over drain in the knee joint .compression

\section{Results}

The patients were clinically and radiologically evaluated at $\mathbf{6}$ and $\mathbf{1 2}$ weeks, $\mathbf{6}$ months. 1 year and $\mathbf{2}$ year postoperative. Clinical evaluation was performed using the International Knee Documentation Committee Score IKDC. Radiological evaluation was performed using computed axial tomography $\mathbf{C T}$ scan to evaluate graft incorporation and healing.

Results according to IKDC score is listed in table $\mathbf{1 .}$

\begin{tabular}{|l|l|l|}
\hline Class & Patients & Percentage \\
\hline \multicolumn{3}{|c|}{ subjective outcome } \\
\hline
\end{tabular}


SOHAG MEDICAL JOURNAL Comparative study between interference screw fixation and implant free technique

Vol. 21 No.1 Jan 2017

\begin{tabular}{|c|c|c|}
\hline Normal A & 19 & $63.33 \%$ \\
\hline Near normal B & 8 & $26.67 \%$ \\
\hline Abnormal C & 3 & $10 \%$ \\
\hline \multicolumn{3}{|c|}{ symptoms evaluation } \\
\hline Normal A & 22 & 73.33\% \\
\hline Near normal B & 5 & $16.67 \%$ \\
\hline Abnormal C & 3 & $10 \%$ \\
\hline \multicolumn{3}{|c|}{ Objective outcome after follow up } \\
\hline Normal A & 25 & $83.33 \%$ \\
\hline Near normal B & 3 & $10 \%$ \\
\hline Abnormal C & 2 & $6.67 \%$ \\
\hline \multicolumn{3}{|c|}{ Range of motion of the knee } \\
\hline Normal A & 25 & $83.33 \%$ \\
\hline Near normal B & 3 & $10 \%$ \\
\hline Abnormal C & 2 & $6.67 \%$ \\
\hline \multicolumn{3}{|c|}{ Knee stability } \\
\hline Normal A & 16 & $53.34 \%$ \\
\hline Near normal B & 8 & $26.66 \%$ \\
\hline Abnormal C & 6 & $20 \%$ \\
\hline
\end{tabular}

Table 1: Clinical results according to IKDC of the first group.

Complications of the first group Bone graft was broken intraoperative in three patients while fixing the femoral tunnel and was removed and we used interference screw instead of the bone graft. Two patients had paraesthesia over the anteromedial portion of tibia which improved subsequently. Two patients had superficial infection and was cured by oral antibiotics and dressing. Complications of the second group Postoperative complications were observed in $\mathbf{5}$ patients. One patient developed superficial wound infection, which resolved on treatment with antibiotics. Arthrofibrosis occurred in 4 patients, requiring intensive treatment, in $\mathbf{2}$ cases arthroscopical arthroysis. There were $\mathbf{3}$ late arthroscopies, for arthrolysis to allow full extension

\begin{tabular}{|l|l|l|}
\hline Class & Patients & Percentage \\
\hline \multicolumn{2}{|c|}{ subjective outcome } \\
\hline Normal A & 19 & $63.33 \%$ \\
\hline Near normal B & 8 & $26.67 \%$ \\
\hline Abnormal C & 3 & $10 \%$ \\
\hline \multicolumn{3}{|c|}{ symptoms evaluation } \\
\hline
\end{tabular}


SOHAG MEDICAL JOURNAL implant free technique

Vol. 21 No.1 Jan 2017

Comparative study between interference screw fixation and

\begin{tabular}{|l|l|l|}
\hline Normal A & 22 & $73.33 \%$ \\
\hline Near normal B & 5 & $16.67 \%$ \\
\hline Abnormal C & 3 & $10 \%$ \\
\hline \multicolumn{2}{|c|}{ Objective outcome after follow up } \\
\hline Normal A & 25 & $\mathbf{8 3 . 3 3 \%}$ \\
\hline Near normal B & $\mathbf{3}$ & $\mathbf{1 0 \%}$ \\
\hline Abnormal C Range of motion of the knee \\
\hline \multicolumn{3}{|c|}{$\mid \mathbf{6 . 6 7 \%}$} \\
\hline Normal A & $\mathbf{2 5}$ & $\mathbf{8 3 . 3 3 \%}$ \\
\hline Near normal B & $\mathbf{3}$ & $\mathbf{1 0 \%}$ \\
\hline Abnormal C & 2 & $\mathbf{6 . 6 7 \%}$ \\
\hline \multicolumn{3}{|c|}{ Knee stability } \\
\hline Normal A & 16 & $\mathbf{5 3 . 3 4 \%}$ \\
\hline Near normal B & $\mathbf{2 6 . 6 6 \%}$ \\
\hline Abnormal C & 6 & $20 \%$ \\
\hline
\end{tabular}

Table2: Clinical results according to IKDC of the second group.

\section{Discussion}

The use of implants for ACL graft fixation has simplified ACL

reconstruction and made surgical outcomes more predictable, but it is not benign as previously thought.

It has been associated with complications such as screw osteolysis, allergic reactions, sterile abscesses, ganglion cysts, fibroxanthoma formation and screw migration [8]. There are problems of soft tissue irritation with cortical posts and staples, requiring hardware removal in $\mathbf{2 1 \%}$ of the patients in one series [9]. There is also a risk of graft injury during insertion with metal interference screws [10] ACL reconstruction with autogenous semitendinosus (ST) and gracilis (G) tendons has become a common surgical procedure The advantages of using them are well known; the most important is its relatively low donor site morbidity This graft has an ultimate tensile load reported to be as high as $\mathbf{4 1 0 8} \mathrm{N}$ (twice the strength of the native ACL) [11].as Pavlik A.et al [12]published their results to elucidate the properties of press-fit fixation. They reported that these techniques were simple, cost-effective, and reliable alternatives for graft fixation in ACL reconstruction. The advantages of press-fit fixation were direct bone-tobone healing of the graft, decreased donor site morbidity, and cost effectiveness. In this study we used bone graft harvested from the femoral and tibial site of ACL to impact the hamstring tendon and enhance bone 
SOHAG MEDICAL JOURNAL implant free technique

Vol. 21 No.1 Jan 2017

healing in the tunnel. This technique get benefit from, press fit technique and hamstring tendon harvest which has relatively low morbidity. Mosaicoplasty knifeallows optimum graft size, preserve the graft architecture, excellent adaptation to the defect configuration, however, it has a steep learning curve to handle the knife and harvest the bony graft. We divided our patients in to two group, one group we use the implant free technique and the other group we fix the graft with interference screw and we found that the result of implant free is comparable to the result of interference screw as regard subjective .objective,sympoms and range of motion

\section{Conclusion.}

The implant-free press-fit technique for anterior cruciate ligament reconstruction using hamstring grafts with anatomic graft placement is an innovative technique to preserve the cartilage and meniscal status without significant differences between the operated and non operated knees in the long term. Significantly less pain was noted in the hamstring group, when testing for kneeling and knee walking.

\section{References}

1. Lohmander LS, Englund PM, Dahl LL, RoosEM:The Long-term Consequence of Anterior Cruciate Ligament and Meniscus Injuries:Osteoarthritis.Am J Sports Med 2007,35:1756-1769

2. Taylor DC, Posner M, Curl WW, FeaginJA:Isolated tears of the anterior
Mohamed Ali Ahmed

cruciate ligament: over 30-year followup of patients treated with arthrotomy and primary repair. Am J Sports Med2009,37:65-71

3. Drogset JO, Straume LG, Bjørkmo I, Myhr G:A prospective randomized study of ACL reconstructions using bonepatellar tendon-bone grafts fixed with bio absorbable or metal interference screws. Knee Surg Sports Traumatol Arthrosc2011,19:753-9.

4-.Shelbourne KD, TrumperRV:Preventing anterior knee pain after anterior cruciate ligament reconstruction. Am J Sports

Med1997,25:41-47.

5. Hertel P:Technik der offenenErsatzplastik des vorderenKreuzbandesmitautologerPatell arsehne.AnatomischeRekonstruktion in schraubenfreierPress-fit-Technik.

Arthroskopie1997, 10:240-245.

6. Boszotta $\mathrm{H}$ : Arthroscopic anterior cruciate ligament reconstruction using a patellar tendon graft in press-fit technique: surgical technique andfollowup.Arthroscopy1997, 13:332-339

7. Pavlik A, Hidas P, Czigány T, Berkes I: Biomechanical evaluation of press-fit femoral fixation technique in ACL reconstruction.

Knee

SurgSportsTraumatol Arthrosc2004, 12:528-33

8.Appelt A, Baier M (2007) Recurrent locking of knee joint caused by intraarticular migration of bioabsorbabletibial interference screw after arthroscopic ACL reconstruction. 
SOHAG MEDICAL JOURNAL Comparative study between interference screw fixation and implant free technique

Vol. 21 No.1 Jan 2017

Mohamed Ali Ahmed

Knee Surg Sports TraumatolArthrosc

15:378-380

phosphate screws. Arthroscopy

22:1204-1210

9.Howell SM, Deutsch ML (1999)

11. Gobbi A, Mahajan S, Zanazzo M, Tuy

Comparison of endoscopic and twoincision techniques for reconstructing a torn anterior cruciate ligament using hamstring tendons. Arthroscopy 15:594606

10.Zantop T, Weimann A, Schmidtko R, Herbort M, Raschke M, Petersen W (2006) Graft laceration and pullout strength of soft-tissue anterior cruciate ligament reconstruction: in vitro study comparing titanium, poly-d, l-lactide, and poly-d, 1-lactide-tri-calcium B (2003) Patellar tendon versus quadrupled bone semitendinosus anterior cruciate ligament reconstruction; a prospective clinical investigation in athletes.Arthroscopy 19(6):592-601.

12.10. Pavlik A, Hidas $P$, Tallay A, Toman J, Berkes I (2006) Femoralpress fit fixation technique in anterior cruciate ligament reconstruction using bonepatellar tendon-bone graft: a prospective clinical evaluation of $\mathbf{2 8 5}$ patients. Am J Sports Med 34(2):220-225 